\title{
LONG TERM EFFECTS OF MILITARY SERVICE ON THE DISTRIBUTION OF EARNINGS
}

\author{
by \\ Brigham R. Frandsen * \\ Massachusetts Institute of Technology
}

CES 09-17 August, 2009

The research program of the Center for Economic Studies (CES) produces a wide range of economic analyses to improve the statistical programs of the U.S. Census Bureau. Many of these analyses take the form of CES research papers. The papers have not undergone the review accorded Census Bureau publications and no endorsement should be inferred. Any opinions and conclusions expressed herein are those of the author(s) and do not necessarily represent the views of the U.S. Census Bureau. All results have been reviewed to ensure that no confidential information is disclosed. Republication in whole or part must be cleared with the authors.

To obtain information about the series, see www.ces.census.gov or contact Cheryl Grim, Editor, Discussion Papers, U.S. Census Bureau, Center for Economic Studies 2K130B, 4600 Silver Hill Road, Washington, DC 20233, Cheryl.Ann.Grim@census.gov. 


\begin{abstract}
I estimate the long term effect of military service on quantiles of earnings and education using the Vietnam draft lottery eligibility status as an instrument. I compare the local quantile treatment effect estimator studied by Abadie, Angrist, and Imbens (2002) to the instrumental variables quantile regression technique developed by Chernozhukov and Hansen (2008). Ordinary quantile regression shows a large negative association between service in Vietnam and earnings of white men, with the effect increasing in magnitude for the upper quantiles. Quantile treatment effects estimates show the opposite pattern, although much smaller in magnitude, with a small negative effect at the lower end of the distribution, and a small positive effect at the upper end. This suggests the ordinary quantile result is due to heterogeneous selection effects. The two methods of quantile treatment effects estimation give similar results.
\end{abstract}

* This study was conducted while the author was a Special Sworn Status researcher of the U.S. Census Bureau at the Boston Research Data Center. Any opinions and conclusions expressed herein are those of the author and do not necessarily represent the views of the U.S. Census Bureau. All results have been reviewed to ensure that no confidential information is disclosed. Support for this research at the Boston RDC from NSF (ITR-0427889) is also gratefully acknowledged. 


\section{Introduction}

As the debate over whether to reinstate the military draft continues (Rangel, December 31, 2002; Rangel, 2007; The Wall Street Journal, November 25, 2006), accurate measures of the effects of active duty military service have critical policy implications. Furthermore, the effects of military service may be very different for individuals at different ends of the socio-economic spectrum, and so reinstating the draft could have significant distributional consequences. In this paper I study the effects of military service in Vietnam across the distribution by using draft lottery status as an instrumental variable in a quantile treatment effects framework. I will focus on the long term impacts on earnings and education.

Numerous studies have attempted to document the average effect of military service, with conclusions ranging from benefits to World War II military service to substantial costs to draftees (e.g., Oi, 1967; Rosen and Taubman, 1982). The comparisons in these studies likely suffer from selection bias: those who serve in the military are different to start with in many ways from those who do not serve ${ }^{1}$. Thus instrumental variables (IV) techniques are needed to accurately assess the causal effect of military service. Angrist (1990) uses the draft lottery as an instrumental variable to estimate the effect of military service on earnings a decade and a half or so after the time of service. Angrist finds a substantial negative earnings effect on veterans for white men. His argument that this was primarily due to loss of experience suggests that the observed effect on earnings should decrease over time in a Mincer-type setting. More recent work (Angrist and Chen, 2007) confirms that the average effects have, indeed, decreased over time, suggesting that loss of experience was driving the earlier negative results.

In order to decompose the average effects cited above into effects on the distribution, quantile treatment effects techniques are needed. Abadie (2002) looks at the effect of Vietnam military service on the distribution of earnings a decade or so after service. He finds that the negative average effect from Angrist (1990) appears to be concentrated at the lower end of the distribution, although his sample size does not allow him to reject that this is not the case. My paper builds on this research by using a much larger sample to examine the long term effect of military service on the distribution of earnings and education. If loss-of-experience effects are truly driving the negative short- to mid-term average effect of military service that disappears in the long run, then the long term effect should be zero across the distribution.

Another purpose of the paper is methodological. My empirical example provides an opportunity to compare two recently developed techniques (Chernozhukov and Hansen, 2008; Abadie, Angrist, and Imbens, 2002) for estimating quantile treatment effects. The comparison offers the chance to highlight the relative performance of the two methods. In addition, since they have different interpretations, the two estimators provide slightly different insights into the empirical question at hand.

In the next section I will describe the econometric framework I use, including a brief review of the two methodologies I am using. In Section 3 I describe the data set I use; Section 4 contains the results of the estimation, and Section 5 concludes.

\section{Estimation framework}

The causal effect of military service in Vietnam on the distribution of outcome $Y$ (for example, log weekly wages) can be thought of as the difference between the distributions of potential outcomes $Y_{d}, d \in\{0,1\}$, which I define according to the canonical potential outcomes framework with heterogeneous treatment effects (Angrist, Imbens, and Rubin, 1996). Following the potential outcomes

\footnotetext{
${ }^{1}$ See Seltzer and Jablon (1974) for a study on the effects of selection on mortality rates, for example.
} 
notation, I index the outcome variable by Vietnam veteran status:

$$
\begin{aligned}
& Y_{0} \equiv \text { potential outcome not having served in Vietnam, } \\
& Y_{1} \equiv \text { potential outcome having served in Vietnam. }
\end{aligned}
$$

The starting point for my estimation framework is the following specification for the conditional quantile function of the potential outcomes:

$$
Q_{\tau}\left(Y_{d} \mid X\right)=\alpha(\tau) \cdot d+X^{\prime} \beta(\tau),
$$

where $\alpha(\tau)$ is the coefficient of interest and $X$ contains additional controls (dummies for birth cohort, race, state of birth, etc.). Using the Skorohod representation, we can write $Y_{d}$ in terms of its conditional quantile function:

$$
Y_{d}=\alpha\left(U_{d}\right) \cdot d+X^{\prime} \beta\left(U_{d}\right), U_{d} \mid X \sim \operatorname{Uniform}(0,1) .
$$

The primary issue with estimating (2) directly is that we do not observe $Y_{d}$ for the whole sample, but rather we only observe $Y_{d}$ when $V E T=d$, where $V E T$ is an indicator for Vietnam veteran status. Because $V E T$ is an endogenous choice variable, it is potentially dependent on $U_{d}$, introducing selection effects into the observed $Y=Y_{V E T}$. Standard conditional quantile estimation techniques such as Koenker and Bassett (1978) therefore will not yield consistent estimates of $\alpha(\tau)$. We can formulate the estimation problem in terms of observables by noting that $Y=Y_{1} V E T+Y_{0}(1-V E T)$, and using the Skorohod representation (2), to write

$$
\begin{aligned}
Y & =Y_{1} V E T+Y_{0}(1-V E T) \\
& =\left(\alpha\left(U_{1}\right) \cdot 1+X^{\prime} \beta\left(U_{1}\right)\right) V E T+\left(\alpha\left(U_{0}\right) \cdot 0+X^{\prime} \beta\left(U_{0}\right)\right)(1-V E T) .
\end{aligned}
$$

Rearranging this, and defining $U \equiv U_{1} V E T+U_{0}(1-V E T)$ gives the following structural relationship between outcome $Y$ and regressors:

$$
Y=\alpha(U) V E T+X^{\prime} \beta(U)^{2},
$$

I will call the right hand side of structural relationship (3) the quantile causal response function,

$$
S_{Y}(\tau, V E T, X) \equiv \alpha(\tau) V E T+X^{\prime} \beta(\tau),
$$

which is increasing in $\tau$, and the parameters of which are the same as those of (1). It is worth emphasizing at this point that the quantile causal response function (4) is in general different from the conditional quantile function of observed outcome, $Q_{\tau}(Y \mid V E T, X)$, because of the endogeneity of $V E T$. The quantile causal response function is instead related to the conditional quantile function of the potential outcome:

$$
Q_{\tau}\left(Y_{d} \mid X\right)=S_{Y}(\tau, d, X), d \in\{0,1\} .
$$

\footnotetext{
${ }^{2}$ The following steps of algebra yield the result:$$
Y=Y_{1} V E T+Y_{0}(1-V E T)
$$$$
=\left(\alpha\left(U_{1}\right) \cdot 1+X^{\prime} \beta\left(U_{1}\right)\right) V E T+\left(\alpha\left(U_{0}\right) \cdot 0+X^{\prime} \beta\left(U_{0}\right)\right)(1-V E T)
$$$$
=\alpha\left(U_{1}\right) V E T+X^{\prime} \beta\left(U_{1}\right) V E T+X^{\prime} \beta\left(U_{0}\right)(1-V E T)
$$$$
=\left(\alpha\left(U_{1} V E T+U_{0}(1-V E T)\right) V E T+X^{\prime} \beta\left(U_{1} V E T+U_{0}(1-V E T)\right)\right) V E T
$$$$
+X^{\prime} \beta\left(U_{1} V E T+U_{0}(1-V E T) U_{0}\right)(1-V E T)
$$$$
=\left(\alpha(U) V E T+X^{\prime} \beta(U)\right) V E T+X^{\prime} \beta(U)(1-V E T)
$$$$
=\alpha(U) V E T+X^{\prime} \beta(U),
$$

where in the first line I used the definition of the potential outcomes $Y_{d}$, in the second line I used the Skorohod representation 2 , and in the second to last line I introduced $U \equiv U_{1} V E T+U_{0}(1-V E T)$. 
This makes it clear that the effect of interest, $\alpha(\tau)$, the difference in the $\tau$-th quantile of the marginal distributions of the potential outcomes, is indeed the treatment effect associated with military service in Vietnam from (4):

$$
\begin{aligned}
Q_{\tau}\left(Y_{1} \mid X\right)-Q_{\tau}\left(Y_{0} \mid X\right) & =S_{Y}(\tau, 1, X)-S_{Y}(\tau, 0, X) \\
& =\alpha(\tau) \cdot 1+X^{\prime} \beta(\tau)-\left[\alpha(\tau) \cdot 0+X^{\prime} \beta(\tau)\right] \\
& =\alpha(\tau) .
\end{aligned}
$$

There are two recently developed frameworks for estimating quantile causal response functions such as (4). Abadie, Angrist, and Imbens (2002) illustrates one which estimates a quantile-specific local treatment effect, which I will refer to as LQTE. A framework for estimating the overall quantile treatment effect by instrumental variables quantile regression (IVQR) is described in Chernozhukov and Hansen (2008). Because the assumptions required for each method, as well as the interpretation of the respective estimates differ, I will briefly review each framework, and discuss the applicability of each framework to my empirical question.

\subsection{Local Quantile Treatment Effect}

In this section I will briefly outline the LQTE framework as developed in more detail in Abadie, Angrist, and Imbens (2002), and discuss how applicable the framework is to the empirical question at hand. I will then describe the estimation and inference procedure.

\subsubsection{LQTE Econometric Framework}

The starting point for LQTE is the insight that for the subpopulation of compliers - those individuals for whom $V E T_{1}>V E T_{0}$ - under certain assumptions, $V E T$ is independent of $Y_{0}$ and $Y_{1}$. These assumptions are that for almost all values of $X$ we have the following:

1. Independence: $\left(Y_{0}, Y_{1}, V E T_{1}, V E T_{0}\right)$ is jointly independent of $E L I G I B L E$ given $X$.

2. Nontrivial Assignment: $\operatorname{Pr}(E L I G I B L E=1 \mid X) \in(0,1)$.

3. First-Stage: $E\left[V E T_{1} \mid X\right] \neq E\left[V E T_{0} \mid X\right]$.

4. Monotonicity: $\operatorname{Pr}\left(V E T_{1} \geq V E T_{0} \mid X\right)=1$.

Since draft lottery number is assigned randomly, and eligibility status is a function of year of birth and lottery number only, the first assumption holds as long as $X$ contains dummies for year of birth and eligibility status doesn't affect the potential outcomes through any channel other than service in Vietnam. This could be problematic if, for example, individuals who were draft eligible were more likely to stay in school to avoid the draft. However, Angrist and Krueger (1995) show that there is little evidence of education-related draft avoidance behavior, so the first assumption seems plausible. The second assumption of nontrivial assignment holds for the birth cohorts I will study, 1950-1952, where the RSN ceilings were 195, 125, and 95, respectively. The high correlation between VET and ELIGIBLE documented in Angrist and Chen (2007), and reproduced later in this paper, is strong evidence for the third assumption. Since the fourth assumption, monotonicity, involves comparisons of counterfactuals, I cannot show direct evidence, but it seems reasonable that being draft eligible would almost surely make an individual weakly more likely to have served in Vietnam. The possibly strong fourth assumption notwithstanding, as a whole these assumptions seem reasonable for my empirical problem.

These assumptions and their implication that for compliers $V E T$ is independent of potential outcomes allow us to overcome the selection effect that made estimation of (4) by ordinary quantile regression techniques inconsistent. In other words, for compliers, the quantile causal response 
function, (4), is equal to $Q_{\tau}\left(Y \mid V E T, X, V E T_{1}>V E T_{0}\right)$, the conditional quantile function:

$$
\begin{aligned}
S_{Y \mid V E T_{1}>V E T_{0}}(\tau, V E T, X) & = \\
Q_{\tau}\left(Y \mid V E T, X, V E T_{1}>V E T_{0}\right) & \equiv \gamma(\tau) V E T+X^{\prime} \lambda(\tau),
\end{aligned}
$$

where as shown in Bassett and Koenker (1982) the parameters of the conditional quantile function can be written as:

$$
\begin{aligned}
& (\gamma(\tau), \lambda(\tau))= \\
& \arg \min _{\gamma, \lambda} E\left[\rho_{\tau}\left(Y-\gamma V E T-X^{\prime} \lambda\right) \mid V E T_{1}>V E T_{0}\right],
\end{aligned}
$$

for the check function $\rho_{\tau}(u) \equiv(\tau-1[u<0]) \cdot u$. If we were able to restrict the sample to compliers, then the solution to the empirical analog of the conditional minimization problem 5 would be a consistent estimator for the parameters in the causal relationship I'm interested in, (4). Since we can't observe the subpopulation of compliers directly, LQTE takes advantage of Abadie's (2000) insight that the conditional expection in (5) can be converted into an unconditional weighted expectation using weights defined by

$$
\begin{aligned}
& \kappa(V E T, E L I G I B L E, X) \equiv \\
& 1-\frac{V E T(1-E L I G I B L E)}{1-\pi_{0}(X)}-\frac{(1-V E T) E L I G I B L E}{\pi_{0}(X)},
\end{aligned}
$$

where $\pi_{0}(X)=\operatorname{Pr}(E L I G I B L E=1 \mid X)$. The key result in Abadie (2000) that LQTE uses is that under the basic assumptions above, for any function $h(Y, V E T, X)$ of the data, the following holds:

$$
\begin{aligned}
& E\left[h(Y, V E T, X) \mid V E T_{1}>V E T_{0}\right]= \\
& \frac{1}{P\left(V E T_{1}>V E T_{0}\right)} E[\kappa h(Y, V E T, X)] .
\end{aligned}
$$

This gives us a feasible way to solve (5), and thus estimate the parameters of (4) for compliers:

$$
\begin{aligned}
\left.(\alpha(\tau), \beta(\tau))\right|_{\text {compliers }} & =\left.(\gamma(\tau), \lambda(\tau))\right|_{\text {compliers }} \\
& =\arg \min _{\gamma, \lambda} E\left[\kappa \rho_{\tau}\left(Y-\gamma V E T-X^{\prime} \lambda\right)\right] .
\end{aligned}
$$

Thus in a setting with a heterogeneous effect of service in Vietnam, the unconditional program (6) suggests how to consistently estimate a quantile-specific local treatment effect for the subpopulation of compliers using only observables. In the next section I will discuss the procedure for solving (6) and performing inference.

\subsubsection{LQTE Estimation and Inference Procedure}

The population estimation program (6) in Section 2.1.1 suggests using the empirical analog to obtain consistent estimates of the structural parameters in (4):

$$
(\hat{\alpha}(\tau), \hat{\beta}(\tau))=\arg \min _{\alpha, \beta} \sum_{i=1}^{N} \hat{\kappa}_{i} \rho_{\tau}\left(Y_{i}-\alpha V E T_{i}-X_{i}^{\prime} \beta\right),
$$

where

$$
\hat{\kappa}_{i}=1-\frac{V E T_{i}\left(1-E L I G I B L E_{i}\right)}{1-\hat{\pi}\left(X_{i}\right)}-\frac{\left(1-V E T_{i}\right) E L I G I B L E_{i}}{\hat{\pi}\left(X_{i}\right)}
$$


with $\hat{\pi}\left(X_{i}\right)$ being a nonparametric estimator of $\operatorname{Pr}\left(E L I G I B L E_{i}=1 \mid X_{i}\right)$. However, when $V E T_{i} \neq$ $E L I G I B L E_{i}, \hat{\kappa}_{i}$ is negative, resulting in a nonconvex minimization problem. I follow Abadie, Angrist, and Imbens's (2002) suggestion to take the expection of $\kappa$ conditional on $U=(Y, V E T, X)$ :

$$
\kappa_{v}=E[\kappa \mid U]=1-\frac{V E T\left(1-v_{0}(U)\right)}{1-\pi_{0}(X)}-\frac{(1-V E T) v_{0}(U)}{\pi_{0}(X)},
$$

where $v_{0}(U)=E[E L I G I B L E \mid U]=\operatorname{Pr}(E L I G I B L E=1 \mid Y, V E T, X)$. Abadie, Angrist, and Imbens (2002) show that $\kappa_{v}$ is non-negative, making computation much more tractable. The estimates can then be found by solving

$$
(\hat{\alpha}(\tau), \hat{\beta}(\tau))=\arg \min _{\alpha, \beta} \sum_{i=1}^{N} \hat{\kappa}_{v, i} \rho_{\tau}\left(Y_{i}-\alpha V E T_{i}-X_{i}^{\prime} \beta\right),
$$

where $\hat{\kappa}_{v}$ is an estimate of $\kappa_{v}$ obtained as shown below.

With this modification to the problem, I followed the following procedure to obtain estimates for each $\tau$ :

1. Nonparametrically estimate $\hat{\pi}_{0}\left(X_{i}\right)=\widehat{\operatorname{Pr}}\left(E L I G I B L E_{i}=1 \mid X_{i}\right)$. Since conditional on year of birth, $E L I G I B L E_{i}$ is random, I estimated this by a simple regression of ELIGIBLE $E_{i}$ on dummies for year of birth.

2. Nonparametrically estimate $\hat{v}_{0}\left(U_{i}\right)=\widehat{\operatorname{Pr}}\left(E L I G I B L E_{i}=1 \mid Y_{i}, V E T_{i}, X_{i}\right)$. Again, the only covariate in $X_{i}$ that needs to be conditioned on is year of birth $(Y O B)$, so I performed series estimation in $V E T \times Y O B$ cells by regressing $E L I G I B L E$ on $Y O B, V E T$, and powers of $Y$, fully interacting everything. I chose the degree, $K$, of powers in $Y$ by minimizing Mallows's (1973) criterion for series estimation:

$$
K=\arg \min _{k} \frac{(E L I G I B L E-\hat{g}(k))^{\prime}(E L I G I B L E-\hat{g}(k))}{N}+2 \hat{\sigma}^{2} \frac{k}{N},
$$

where $Y-\hat{g}(k)$ is the estimated residual from a regression of ELIGIBLE on the full set of interacted covariates with $k$ powers of $Y$, and $\hat{\sigma}^{2}$ is an estimate of the variance of the disturbance using some fixed $\bar{K}$.

3. Construct $\hat{\kappa}_{v, i}=1-\frac{V E T_{i}\left(1-\hat{v}_{0}\left(U_{i}\right)\right)}{1-\hat{\pi}_{0}\left(X_{i}\right)}-\frac{\left(1-V E T_{i}\right) \hat{v}_{0}\left(U_{i}\right)}{\hat{\pi}_{0}\left(X_{i}\right)}$, trim the negative values (which will occur in finite sample), and use it as the weight in a standard weighted quantile regression of $Y$ on $V E T$ and $X$ to get the point estimate $\hat{\alpha}(\tau)$.

The usual standard errors reported with the weighted quantile regression in step 3 cannot be used for inference, because they do not take into account the first step estimation of $\hat{\kappa}_{v}$. Instead I compute the asymptotic variance estimator in Abadie, Angrist, and Imbens (2002). If we define $W=\left[\begin{array}{ll}V E T & X\end{array}\right]$, and $\delta(\tau)^{\prime}=\left(\alpha(\tau), \beta(\tau)^{\prime}\right)$, then the asymptotic variance of $\sqrt{N}(\hat{\delta}(\tau)-\delta(\tau))$ is:

$$
\hat{\Omega}=\hat{J}^{-1} \hat{\Sigma} \hat{J}^{-1},
$$


where

$$
\begin{aligned}
\hat{J}= & \frac{1}{N} \sum_{i=1}^{N} \hat{\kappa}_{v, i} \varphi_{h, i}(\hat{\delta}(\tau)) W_{i} W_{i}^{\prime}, \\
\varphi_{h, i}(\delta)= & \frac{1}{h} \varphi\left(\frac{Y_{i}-W_{i}^{\prime} \delta}{h}\right), \\
\hat{\Sigma}= & \frac{1}{N} \sum_{i=1}^{N} \hat{\psi}_{i} \hat{\psi}_{i}^{\prime}, \\
\hat{\psi}_{i}= & \hat{\kappa}_{i} \cdot\left(\tau-1\left\{Y_{i}-W_{i}^{\prime} \hat{\delta}(\tau)<0\right\}\right) \cdot W_{i}+\hat{H}_{i} \cdot\left\{E L I G I B L E_{i}-\hat{\pi}\left(X_{i}\right)\right\}, \\
\hat{H}_{i}= & \frac{1}{N_{l}} \sum_{i_{l}=1}^{N_{l}}\left(\tau-1\left\{Y_{i l}-W_{i l}^{\prime} \hat{\delta}(\tau)<0\right\}\right) \cdot W_{i l} \\
& \cdot\left(\frac{\left(1-V E T_{i l}\right) E L I G I B L E_{i l}}{\left(\hat{\pi}\left(X_{i}\right)\right)^{2}}-\frac{V E T_{i l}\left(1-E L I G I B L E_{i l}\right)}{\left(1-\hat{\pi}\left(X_{i}\right)\right)^{2}}\right),
\end{aligned}
$$

for observation $i$ in cell $l$ of $X$ with $N_{l}$ observations in the cell. I chose the kernel function $\varphi(\cdot)$ to be the Quartic kernel:

$$
\varphi(u)=\frac{15}{16} \frac{1}{h}\left(1-\left(\frac{u}{h}\right)^{2}\right)^{2} 1\left\{\left|\frac{u}{h}\right|<1\right\}
$$

with the bandwidth $h$ chosen by a simple rule of thumb:

$$
h=N^{-\frac{1}{5}} \hat{\sigma}_{u} .
$$

\subsection{Instrumental Variables Quantile Regression}

In this section I will briefly set up the IVQR framework as illustrated in Chernozhukov and Hansen (2008) in terms of my empirical question, and discuss how plausible the required assumptions might be in this context. I will also outline the estimation and inference procedure.

\subsubsection{IVQR Econometric Framework}

As for LQTE, the IVQR framework starts with the specification of the quantile causal response function in (4), $S_{Y}(\tau, V E T, X)$, and recognizes that because of endogeneity the parameters of this object cannot be estimated by ordinary conditional quantile regression techniques. Under a set of assumptions, a population moment condition is implied that can be used to estimate the structural parameters. These assumptions, described in Chernozhukov and Hansen (2005), are the following:

1. Potential Outcomes: conditional on $X=x$, for each $d \in\{0,1\}, Y_{d}=S_{Y}\left(U_{d}, d, x\right)$, where $S_{Y}(\tau, d, x)$ is strictly increasing in $\tau$ and $U_{d} \sim U(0,1)$.

2. Independence: conditional on $X=x,\left\{U_{d}\right\}$ are independent of ELIGIBLE.

3. Selection: $V E T=\delta(E L I G I B L E, X, V)$ for some unknown function $\delta$ and random vector $V$.

4. Rank Invariance or Rank Similarity: conditional on $X=x, Z=z$,

(a) $\left\{U_{d}\right\}$ are equal to each other; or, more generally,

(b) $\left\{U_{d}\right\}$ are identically distributed, conditional on $V$.

5. Observed Variables consist of $Y, V E T, X$, and $Z$. 
Assumptions 1, 2, 3, and 5 are simply restatements of the treatment effects framework I set up at the beginning of Section 2. The strongest assumption is Assumption 4. Applied to the empirical question at hand, this assumption says roughly that those who would be highly ranked earners (if earnings is the outcome of interest) without having served in Vietnam would also be highly ranked earners if they had served in Vietnam, or at least the deviation in rank across the two states is not systematic. While conditioning on the proper covariates makes this assumption more plausible, it is easy to imagine that ranks across potential Vietnam veteran status could differ in systematic ways that I am not controlling for. Indeed, one motivation for using instrumental variables techniques is that we cannot rule out omitted variables bias. Thus going forward we bear in mind that this assumption may be quite strong for my application.

If we accept the assumptions, the initial insight is that model (4) and the assumption that $E L I G I B L E$ and $X$ are exogenous implies the following moment condition:

$$
\operatorname{Pr}\left(Y \leq S_{Y}(\tau, V E T, X) \mid E L I G I B L E, X\right)=\tau
$$

or rearranging,

$$
\operatorname{Pr}\left(Y-S_{Y}(\tau, V E T, X) \leq 0 \mid E L I G I B L E, X\right)=\tau .
$$

In other words, 0 is the $\tau$ th quantile of $Y-S_{Y}(\tau, V E T, X)$ conditional on ELIGIBLE and $X$, which implies that the parameters of the quantile causal response function (4) satisfy:

$$
\begin{aligned}
0 & =\arg \min _{\gamma} E\left[\rho_{\tau}\left(Y-S_{Y}(\tau, V E T, X)-\gamma E L I G I B L E\right)\right] \\
& =\arg \min _{\gamma} E\left[\rho_{\tau}\left(Y-\alpha(\tau) V E T-X^{\prime} \beta(\tau)-\gamma E L I G I B L E\right)\right] .
\end{aligned}
$$

Written this way, we see that the causal parameter of interest, $\alpha(\tau)$, can be implicitly defined as a solution to an inverse quantile regression problem:

$$
\alpha(\tau) \in\{\alpha \mid \gamma(\alpha, \tau)=0\}
$$

where

$$
\gamma(\alpha, \tau) \equiv \underset{\gamma}{\operatorname{argmin}} E\left[\rho_{\tau}\left(Y-\alpha V E T-X^{\prime} \beta-\gamma E L I G I B L E\right)\right]
$$

is simply the coefficient on $E L I G I B L E$ from an ordinary quantile regression of $Y-\alpha V E T$ on $X$ and $E L I G I B L E$, for some chosen value $\alpha$. If the solution to $(8)$ is unique, the $\tau$-th quantile treatment effect is simply the $\alpha$ such that $\gamma(\alpha, \tau)=0$.

\subsubsection{IVQR Estimation and Inference Procedure}

The IVQR framework outlined in the previous section suggests the following as an empirical estimator for the quantile treatment effect:



where

$$
\hat{\gamma}(\alpha, \tau) \equiv \underset{\gamma}{\operatorname{argmin}} \frac{1}{N} \sum_{i=1}^{N} \rho_{\tau}\left(Y_{i}-\alpha V E T_{i}-X_{i}^{\prime} \beta-\gamma E L I G I B L E_{i}\right)
$$

is the estimated coefficient from the finite-sample quantile regression of $Y-\alpha V E T$ on $X$ and $E L I G I B L E$, and $\operatorname{Var} \widehat{(\hat{\gamma}(\alpha}, \tau))$ is a consistent estimator for the variance of $\hat{\gamma}(\alpha, \tau)$. The key advantage of this approach is that the objective function in $10 p, W_{n}(\alpha, \tau) \equiv \frac{\hat{\gamma}(\alpha, \tau)^{2}}{\operatorname{Var}(\hat{\gamma}(\alpha, \tau))}$, is simply the test statistic for testing the null hypothesis $\hat{\gamma}(\alpha, \tau)=0$. The so-called dual inference on $\hat{\alpha}(\tau)$ is 
therefore very straightforward: a level- $p$ confidence interval for $\alpha(\tau)$ contains those values of $\alpha$ for which we do not reject the null hypothesis $\hat{\gamma}(\alpha, \tau)=0$ at level $p$ :

$$
C R_{p}[\alpha(\tau)]=\left\{\alpha: W_{n}(\alpha, \tau)<c_{p}\right\} .
$$

In summary, then, for any $\tau$ a simple procedure for estimation and inference in the IVQR framework is the following:

1. Choose a grid of candidate values for $\hat{\alpha}(\tau):\left\{\alpha_{j}, j=1, \ldots, J\right\}$, and run ordinary quantile regression of $Y_{i}-\alpha_{j} V E T_{i}$ on $X_{i}$ and $E L I G I B L E_{i}$ to get coefficients $\hat{\beta}\left(\alpha_{j}, \tau\right)$ and $\hat{\gamma}\left(\alpha_{j}, \tau\right)$.

2. Perform the test of the null hypothesis $\hat{\gamma}\left(\alpha_{j}, \tau\right)=0$, and save the test statistic $W_{n}\left(\alpha_{j}, \tau\right)$ and the $p$-value.

3. Choose $\hat{\alpha}(\tau)$ as the $\alpha_{j}$ that minimizes $W_{n}\left(\alpha_{j}, \tau\right)$.

4. By the dual inference principle, a level- $p$ confidence interval for $\hat{\alpha}(\tau)$ can be constructed from those values of $\alpha_{j}$ such that $W_{n}\left(\alpha_{j}, \tau\right)$ has a $p$-value greater than $p$.

In my implementiation, I chose the grid to be centered around the 2SLS estimate of the treatment effect, extending four standard deviations in each direction. I set the fineness of the grid to give 20 equally spaced points. Where the estimated confidence interval reached an endpoint of the grid, I extended the grid in that direction until the confidence interval was covered. The quantile regression and calculation of the test statistics for dual inference was done using standard Stata commands.

In contrast to the LQTE framework, the assumptions of which seem quite plausible for this setting, but which gives only a local effect for the compliers, the IVQR framework estimates the quantile treatment effect for the population as a whole, but at the cost of the fairly strong Assumption 4 .

\section{Data}

In order to use draft lottery eligibility status as an instrument for military service, I require the exact date of birth. Although this is confidential data, I have obtained permission as a Special Sworn Status employee of the Census Bureau's Center for Economic Studies to analyze the same non-public one-in-six 2000 U.S. census file used by Angrist and Chen (2007). The sample I analyze consists of men born in the 50 states or Washington, D.C. between 1950 and 1952. The sample size is 696,530 white men, and 96,217 non-white men. About 24 percent of both whites and non-whites served in the military during the Vietnam era. Draft eligibility status is constructed by matching date of birth with assigned lottery numbers and draft eligibility ceilings from the Selective Service System. About 38 percent of the sample was draft eligible. As in Angrist and Chen (2007), I construct a variable for log weekly wages by dividing wage and salary income by weeks worked and taking logs. Also as in Angrist and Chen (2007), for a measure of education, I use Jaeger's (1997) method for imputing highest grade completed from the 2000 education recode variable. The covariates I use are indicators for year of birth, month of birth, and state of birth. I perform the analyses separately for white and non-white men.

Table 1. reproduced from Table 1 in Angrist and Chen (2007), reports summary statistics on the variables I use in the analysis, including comparisons of veterans and non-veterans by race. The table shows in very rough terms what the OLS analysis in Section 4 will show more precisely. A simple comparison of means shows that white veterans earn on average substantially less than non-veterans, with the average earnings for white veterans being $\$ 39,472$, and the average earnings for white non-veterans being $\$ 48,553$. For non-whites, on the other hand, veterans earn somewhat more on average $-\$ 28,505$ - than non-veterans, who earn on average $\$ 27,287$. The same pattern 
Table 1: Means of selected variables, by race and veteran status, men born 1950-52

\begin{tabular}{|c|c|c|c|}
\hline & $\begin{array}{l}\text { All } \\
(1)\end{array}$ & $\begin{array}{c}\text { Vietnam veteran } \\
(2)\end{array}$ & $\begin{array}{c}\text { Non-veteran } \\
(3)\end{array}$ \\
\hline \multicolumn{4}{|c|}{ A. Whites } \\
\hline Draft eligibility (by RSN) & .376 & .532 & .327 \\
\hline Veteran status (served in Vietnam Era) & .236 & 1 & 0 \\
\hline Wage and salary income & $\$ 46,406$ & $\$ 39,472$ & $\$ 48,553$ \\
\hline Log weekly earnings (positive values) & 6.75 & 6.65 & 6.78 \\
\hline Imputed highest grade completed & 13.8 & 13.4 & 13.9 \\
\hline $\mathrm{N}$ & 696,530 & 166,652 & 529,878 \\
\hline \multicolumn{4}{|c|}{ B. Nonwhites } \\
\hline Draft eligibility (by RSN) & .382 & 482 & .350 \\
\hline Veteran status (served in Vietnam Era) & .244 & 1 & 0 \\
\hline Wage and salary income & $\$ 27,584$ & $\$ 28,505$ & $\$ 27,287$ \\
\hline Log weekly earnings (positive values) & 6.41 & 6.43 & 6.41 \\
\hline Imputed highest grade completed & 12.6 & 13.0 & 12.4 \\
\hline $\mathrm{N}$ & 96,217 & 23,246 & 72,971 \\
\hline
\end{tabular}

Note: Sample weights are used in all estimates and statistics.

holds for highest grade completed. White veterans completed on average 13.4 years of schooling, compared to 13.9 years for white non-veterans, while non-white veterans completed 13.0 years of schooling, compared to 12.4 for non-white non-veterans.

As I discussed in Section 2, the draft eligibility status is an attractive candidate for an instrument for military service because conditional on year of birth it is randomly assigned, and it is correlated with military service. The results of first stage regressions of an indicator for service in Vietnam on draft eligibility and covariates that show this correlation are reported in Table 2.

The results, which can also be found in Angrist and Chen (2007), show that whites who were draft eligible are 14.5 percent more likely to have served in the military, while non-whites who were draft eligible are 9.4 percent more likely to have served in the military. The effect of draft eligibility on the probability of military service can be seen even more strikingly in Figure 1. reproduced from Figure 1 in Angrist and Chen (2007), which shows the probability of being a veteran by draft lottery number for each cohort I am analyzing, as well as for the 1953 birth cohort for comparison. For each cohort I am analyzing, there is a sharp drop in veteran status at the respective eligibility ceilings, which was 195 for the 1950 cohort, 125 for the 1951 cohort, and 95 for the 1952 cohort. Draft eligibility, therefore, appears to be a strong instrument for military service in Vietnam.

\section{Results}

In this section, I will present the results of estimating specifications in the form of model (4). As a baseline, I will start with non-quantile results of OLS and two-stage least squares, followed by ordinary quantile regression results. I will then present results from the two methods of quantile treatment effects. 

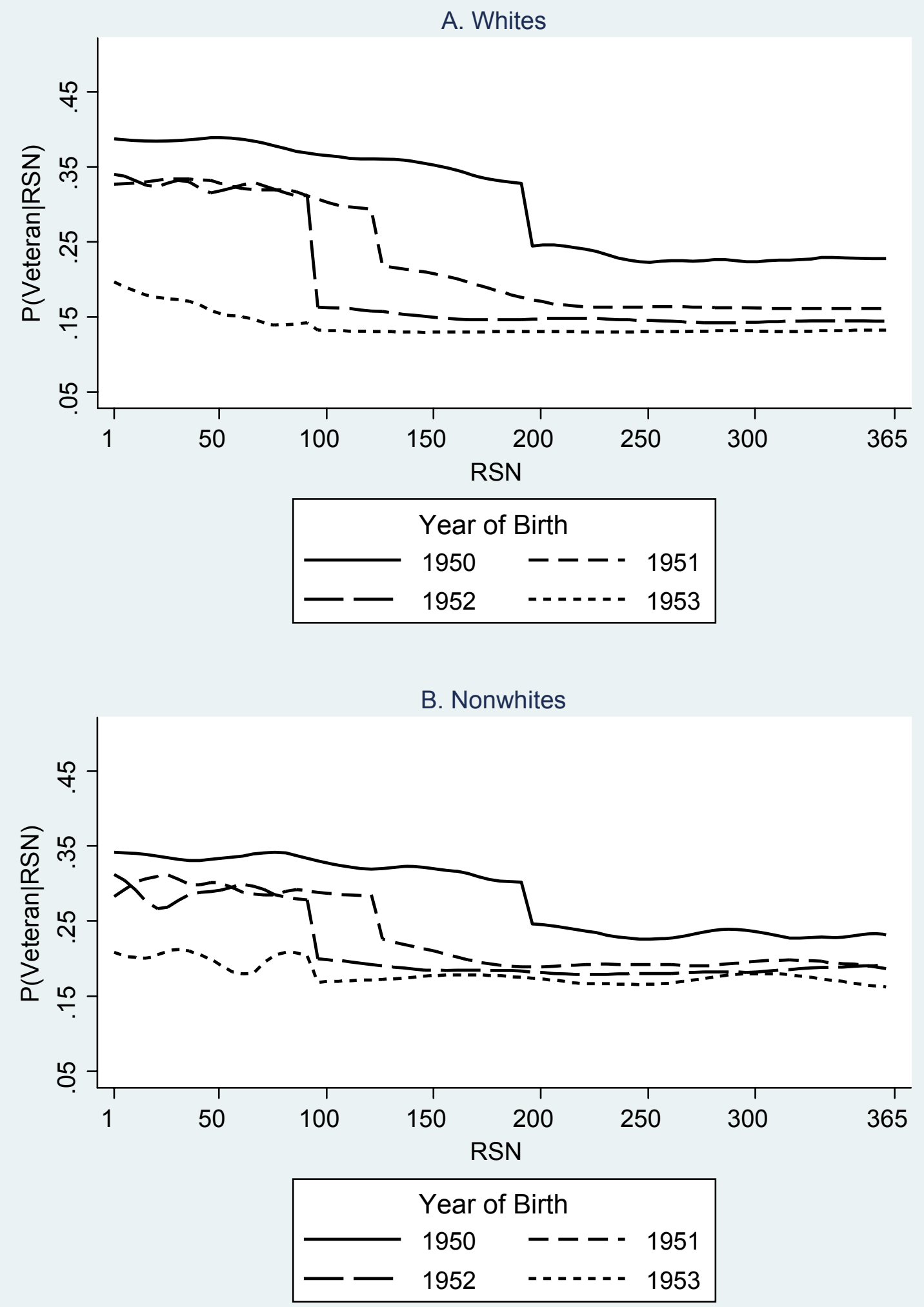

Figure 1: Probability of military service in Vietnam, by race and birth cohort. The sample is US born men, birth year indicated. 
Table 2: First stage, by race, US born men, born 1950-1952

\begin{tabular}{lcc}
\hline & Whites & .094 \\
\cline { 3 - 3 } $\begin{array}{l}\text { Draft-eligibility } \\
\text { effect }\end{array}$ & .145 & $(.0034)$ \\
& & \\
\hline Note: This table reports the coefficient on an indicator \\
for draft eligibility from a regression of an indicator for \\
military service in Vietnam on draft eligibility, as well as \\
dummies for year of birth, month of birth, and state of \\
birth. The sample consists of men born in the US between \\
1950 and 1952.
\end{tabular}

\subsection{OLS, Two-stage Least Squares, and Quantile Regression}

The results of estimating a non-quantile version of model (4) are presented in Table 3 The OLS results in column (1), panel A, reflect the negative correlation between military service and earnings for white men, with a coefficient on service in Vietnam of -.1207 (.0026). The two-stage least squares estimate of the effect of military service in column (2), however, is much smaller, at -.0038 (.0163), and is not significantly different from zero, implying that the negative OLS relationship is due to selection effects. For non-whites, however, column (1) in panel B shows a significant positive association between log weekly wages and service in Vietnam, with a point estimate of .028 (.0074). Once again, however, when the selection effect is controlled for using two-stage least squares, column (2) shows an insignificant negative effect, -.037 (.067), although the standard error is quite large. The same qualitative results hold for wage and salary income, shown in the second row of Table 3. Education, however, has quite a different association with Vietnam service. While OLS shows a coefficient of $-.549(.0075)$ on veteran status, the 2SLS result in column (2) shows a significant positive average effect on military service on education, at .345 (.0540). For non-whites, both OLS and 2SLS show a positive relationship between highest grade completed and veteran status, but the 2SLS estimate is not significantly different from zero. Veterans' access to education through the GI Bill likely contributes to this result.

Columns (3) through (5) of Table 3 show the coefficients on an indicator for military service from ordinary conditional quantile regressions of the outcomes. The results decompose the OLS correlation reported in column (1) into quantiles, but do not take into account the endogeneity of service in Vietnam. For white men, we see service in Vietnam is associated with a .059 decrease in $\log$ weekly wages at the 25 th percentile, a .09 decrease at the median, and a .145 decrease at the 75 th percentile. Thus service in Vietnam is associated with a larger (in magnitude) negative association with $\log$ weekly wages at the higher conditional quantiles. Figure 2 plots the coefficient on $V E T$ from a quantile regression of $\log$ weekly wages for white men by quantile. The figure shows pattern evident in Table 3. with a negative association across the distribution, but increasingly negative for higher quantiles. Thus Vietnam veteran status appears to have a location-scale association with log weekly earnings, with the an overall downward shift in location, and a compressing effect on the distribution of log wages.

There are several possible interpretations of this pattern across the conditional distributions. One is that the negative selection effect is even more pronounced at higher quantiles. An economic explanation for this is that the opportunity cost of military service is higher at higher quantiles, and therefore negative selection is stronger than at the lower end of the distribution, although even at the lower quantiles there is a negative association between service and log wages. Another interpretation is that military service contributes to wage compression. The loss-of-experience explanation given 
Table 3: OLS, Two-stage least squares, and ordinary quantile regression estimates

\begin{tabular}{|c|c|c|c|c|c|}
\hline \multirow[b]{2}{*}{ Dependent variable } & \multirow[b]{2}{*}{$\begin{array}{c}\text { OLS } \\
(1)\end{array}$} & \multirow[b]{2}{*}{$\begin{array}{c}\text { 2SLS } \\
(2)\end{array}$} & \multicolumn{3}{|c|}{ Quantile regression } \\
\hline & & & $\begin{array}{l}.25 \\
(3)\end{array}$ & $\begin{array}{c}.5 \\
(4)\end{array}$ & $\begin{array}{l}.75 \\
(5)\end{array}$ \\
\hline Log weekly wages & $\begin{array}{l}-.1207 \\
(.0026)\end{array}$ & $\begin{array}{c}\text { A. Wl } \\
-.0038 \\
(.0163)\end{array}$ & $\begin{array}{c}-.059 \\
(.0022)\end{array}$ & $\begin{array}{c}-.090 \\
(.0017)\end{array}$ & $\begin{array}{c}-.145 \\
(.0020)\end{array}$ \\
\hline Wage and salary income & $\begin{array}{c}-8616 \\
(161)\end{array}$ & $\begin{array}{c}-517 \\
(1240)\end{array}$ & & & \\
\hline Highest Grade completed & $\begin{array}{c}-.549 \\
(.0075)\end{array}$ & $\begin{array}{c}.345 \\
(.0540)\end{array}$ & & & \\
\hline & & B. Non- & & & \\
\hline Log weekly wages & $\begin{array}{c}.028 \\
(.0074)\end{array}$ & $\begin{array}{l}-.037 \\
(.067)\end{array}$ & $\begin{array}{c}.067 \\
(.0085)\end{array}$ & $\begin{array}{c}.047 \\
(.0062)\end{array}$ & $\begin{array}{c}-.004 \\
(.0064)\end{array}$ \\
\hline Wage and salary income & $\begin{array}{l}1324 \\
(313)\end{array}$ & $\begin{array}{c}3476 \\
(3231)\end{array}$ & $\begin{array}{c}2300 \\
(.000)\end{array}$ & $\begin{array}{l}3794 \\
(225)\end{array}$ & $\begin{array}{l}2416 \\
(280)\end{array}$ \\
\hline Highest Grade completed & $\begin{array}{c}.542 \\
(.0204)\end{array}$ & $\begin{array}{c}.239 \\
(.2316)\end{array}$ & $\begin{array}{c}.620 \\
(.0000)\end{array}$ & $\begin{array}{c}.557 \\
(.1990)\end{array}$ & $\begin{array}{c}.000 \\
(.0000)\end{array}$ \\
\hline
\end{tabular}

Notes: the table contains coefficients on an indicator for service in Vietnam from regressions of the dependent variable shown. All regressions include controls for year of birth, month of birth, and state of birth. An indicator for draft eligibility is used as an instrument for the 2SLS estimates, and service in Vietnam is treated as endogenous. The sample is US born men born 1950-1952. Quantile estimates for outcomes wage and salary income and highest grade completed for white men failed to converge, and are not reported. 




Figure 2: Ordinary Quantile Regression estimates of the effect of military service in Vietnam on log weekly wages. The sample is US born white men, born 1950-1952. 
Table 4: Local Quantile Treatment Effects estimates

\begin{tabular}{|c|c|c|c|c|c|}
\hline \multirow[b]{2}{*}{ Dependent variable } & \multicolumn{5}{|c|}{ LQTE } \\
\hline & $\begin{array}{l}.15 \\
(1)\end{array}$ & $\begin{array}{l}.25 \\
(2)\end{array}$ & $\begin{array}{l}.5 \\
(3) \\
\end{array}$ & $\begin{array}{l}.75 \\
(4)\end{array}$ & $\begin{array}{l}.85 \\
(5)\end{array}$ \\
\hline Log weekly wages & $\begin{array}{c}.000 \\
(.0172)\end{array}$ & $\begin{array}{l}\text { A. Wh } \\
.005 \\
(.0136)\end{array}$ & $\begin{array}{c}.004 \\
(.0114)\end{array}$ & $\begin{array}{c}.000 \\
(.0158)\end{array}$ & $\begin{array}{c}.003 \\
(.0222)\end{array}$ \\
\hline \multirow[b]{2}{*}{ Log weekly wages } & \multicolumn{3}{|c|}{ B. Non-whites } & \multirow[b]{2}{*}{$\begin{array}{c}.001 \\
(.0676)\end{array}$} & \multirow[b]{2}{*}{$\begin{array}{c}-.004 \\
(.0935)\end{array}$} \\
\hline & $\begin{array}{c}-.010 \\
(.0925)\end{array}$ & $\begin{array}{c}.004 \\
(.0741)\end{array}$ & $\begin{array}{c}.013 \\
(.0589)\end{array}$ & & \\
\hline Wage and salary income & $\begin{array}{c}0 \\
(816)\end{array}$ & $\begin{array}{c}2631 \\
(3736)\end{array}$ & $\begin{array}{c}1380 \\
(2013)\end{array}$ & $\begin{array}{c}873 \\
(2172)\end{array}$ & $\begin{array}{c}947 \\
(2589)\end{array}$ \\
\hline Highest Grade completed & $\begin{array}{c}.000 \\
(.0340)\end{array}$ & $\begin{array}{l}.000 \\
(.0310)\end{array}$ & $\begin{array}{c}.557 \\
(.1120)\end{array}$ & $\begin{array}{l}.000 \\
(.0460)\end{array}$ & $\begin{array}{l}.325 \\
(.4400)\end{array}$ \\
\hline \multicolumn{6}{|c|}{$\begin{array}{l}\text { Notes: the table contains coefficients on an indicator for service in Vietnam from regressions of the } \\
\text { dependent variable shown. All regressions include controls for year of birth, month of birth, and } \\
\text { state of birth. An indicator for draft eligibility is used as an instrument and service in Vietnam } \\
\text { is treated as endogenous. The sample is US born men, with year of birth } 1950-1952 \text {. Quantile } \\
\text { estimates for outcomes wage and salary income and highest grade completed for white men failed } \\
\text { to converge, and are not reported. }\end{array}$} \\
\hline
\end{tabular}

by Angrist (1990) and Angrist and Chen (2007) is consistent with this interpretation. Since wage profiles tend to fan out with experience (see, for example, Mincer, 1974), if military service essentially sets individuals back in time by the length of the service, the distribution of earnings will tend to be more compressed, and at a lower level, than without military service. However, since the earnings here are from around three decades after Vietnam, it seems unlikely that experience could account for such a large difference, since the wage-experience profile flattens out over time (see again, for example, Mincer, 1974). The selection effect interpretation seems much more plausible, and the fact that the two-stage least squares estimates in column (2) are not significantly different from zero suggests that this is the case. The quantile treatment effects estimates I will present next take into account the selection effect, and will serve to distinguish between these two interpretations.

\subsection{Quantile Treatment Effects}

In this section I will present the results of estimating model (4) using the LQTE and IVQR methods I discussed above, which combine instrumental variables methods' ability to overcome selection effects with quantile regression. I will start by presenting the results of each method separately, after which I will compare the methods graphically, and discuss possible intrepretations.

Table 4 contains the results of estimating model (4) by LQTE, discussed in Section 2.1. Panel A shows that for whites, the effect on log weekly wages is very close to zero throughout the distribution. The same holds true for log weekly wages of non-whites, shown in Panel B. Likewise, the estimated effect of military service on wage and salary income for non-whites, while slightly positive, never exceeds its standard deviation at any point in the distribution. The last row of Panel B, however, shows that in the middle of the distribution military service had a significant, positive effect on highest grade completed, with an estimated effect of .557 years and a standard deviation of .112. Thus for the subpopulation of compliers, there appears to be little effect of military service on earnings, and around a half-year increase in schooling at the middle of the distribution for nonwhites. 
Table 5 contains the results of estimating model (4) by IVQR, discussed in Section 2.2. Panel A shows the effect of military service on the log weekly wages of white men. At the 15th percentile, the estimated effect is -.020, with a 95 percent confidence interval from -.061 to -.0038. At the median, the estimated effect is zero, with a confidence interval from -.02 to .01. At the 85th percentile, the point estimate is .045 , with a confidence interval from .0044 to .070. Thus here, as well, the effect on log weekly wages of white men is close to zero throughout the distribution, although the estimates are marginally less than zero at the lower end of the distribution and marginally greater than zero at the upper end of the distribution. Figure 3, which I will discuss below, shows that this slight upward trend is indeed (marginally) statisitcally significant at the five percent level. Panel B shows a similar result for non-whites. The 95 percent confidence interval for the effect on log weekly wages and wage and salary income contains zero at every point of the distribution. As long as the assumptions in Section 2.2.1 hold, in particular the rank invariance or rank similarity condition, we can interpret these results to mean that the effect of military service on the quantiles of earnings is quite small for the sample as a whole, as well as for the subsample of compliers. 





Figure 3 illustrates the difference between the ordinary quantile regression results and the QTE results. The figure plots ordinary quantile regression and IVQR point estimates and confidence intervals for the effect of military service on white males' log weekly wages by quantile. The most striking contrast is that the ordinary quantile regression estimates become more and more negative as we move up the distribution, while the quantile treatment effect estimates remain close to zero throughout the distribution. Also, analogously to the relationship between OLS and two-stage least squares estimators, the confidence interval for ordinary quantile regression is much tighter than for the quantile treatment effects estimators.

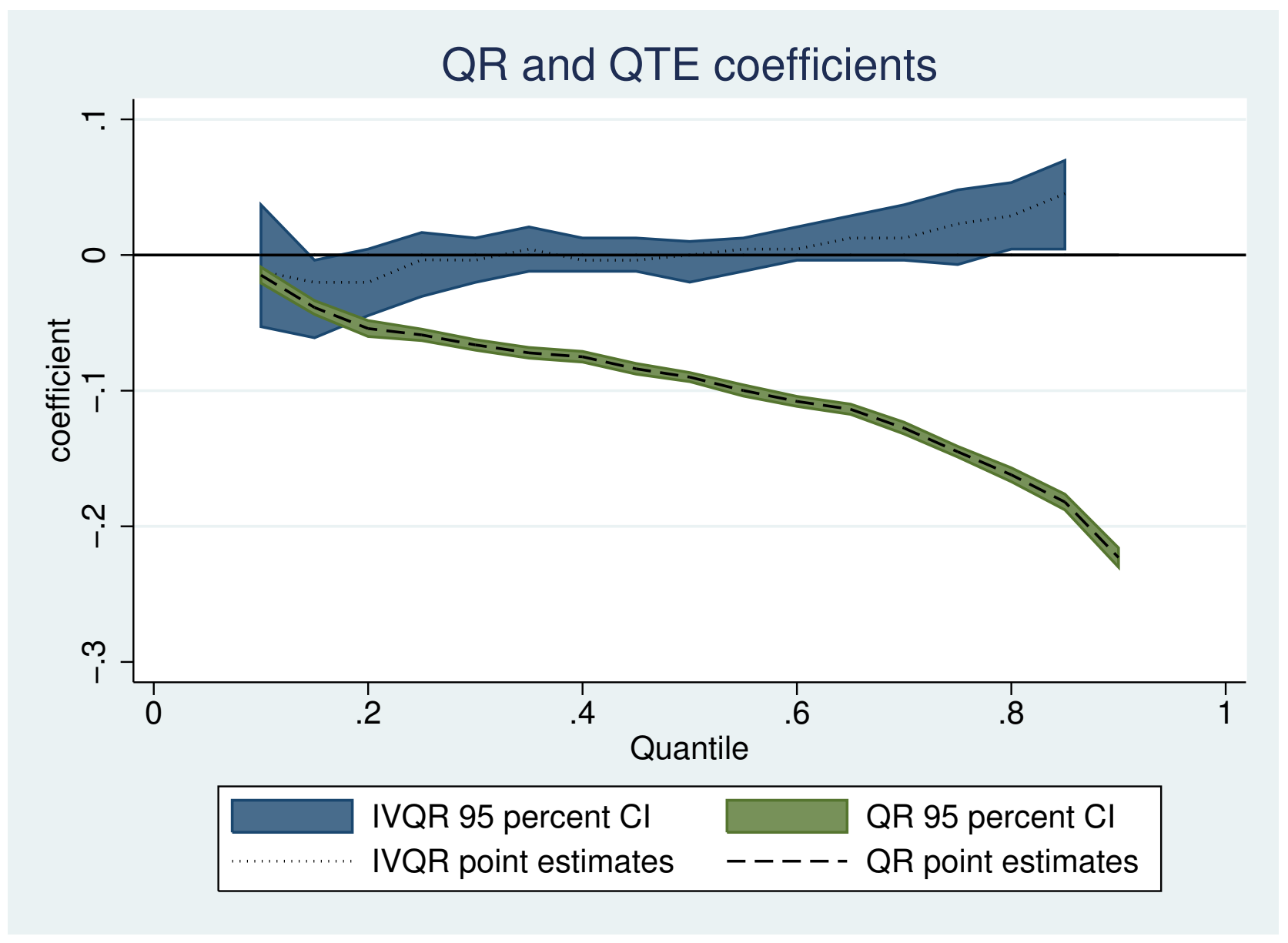

Figure 3: Ordinary quantile regression and Instrumental Variables Quantile Regression estimates of the effect of military service in Vietnam on log weekly wages. The sample is US born white men, born 1950-1952.

In Figure 4 I plot the two quantile treatment effects estimators' point estimates and 95 percent confidence intervals, and we see that they are quite similar. Both show an effect close to zero across the distribution, although the IVQR estimate is marginally significantly less than zero near the 15th percentile, where the point estimate is -.02015, with an upper bound of -.00381, and marginally significantly greater than zero near the 80 th percentile and above, with a point estimate of .02887 , and a lower bound of .00436 . Thus, the IVQR estimates show a slight upward trend in the effect as we move up the distribution, as opposed to the distinct downward trend in the ordinary quantile regression estimates. A final point of comparison is that the IVQR estimates seem to be slightly 


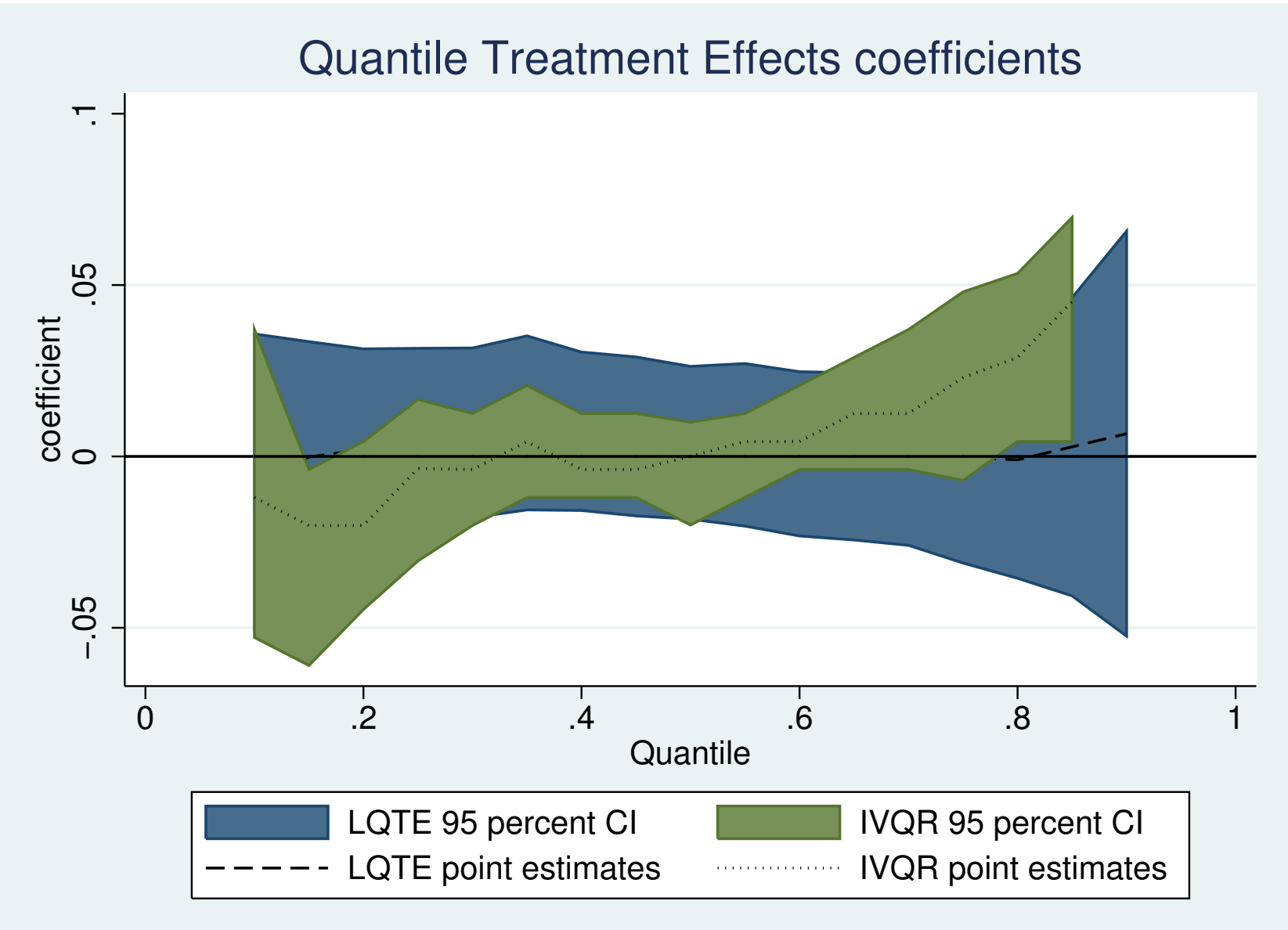

Figure 4: Local Quantile Treatment Effects and Instrumental Variables Quantile Regression estimates of the effect of military service in Vietnam on log weekly wages. The sample is US born white men, born 1950-1952. 
more precise than the LQTE, possibly because LQTE implicitly only estimates the effect for the subpopulation of compliers, which in this sample comprises about $14.5 \%$ of the population (the size of the first stage, reported in Table 2), while IVQR estimates the quantile treatment effect for the whole sample.

Taken together, these results seem to confirm the interpretation that the ordinary conditional quantile results are due to a stronger selection effect at the upper end of the distribution, and that after 30 years loss-of-experience effects are negligible. This supports a Mincer-style interpretation of a wage-experience profile that flattens over time. However, while the loss-of-experience effect seems to have faded away across the distribution, the marginally significant negative effect around the 15th percentile and the positive effect at the 80th percentile as above for the IVQR suggests there is somewhat of a long-term impact at the bottom and top of the distribution, albeit small in magnitude.

\subsection{A Word on Computation in Practice}

Before concluding, I will discuss briefly some observations on computing these estimators in practice. For roughly continuous data, such as log weekly wages, all estimators computed fairly quickly, even for the large sample size. In particular, the minimization problem in the inverse quantile regression procedure within the IVQR framework was very well behaved. Figure 5 plots the objective function in (10), $W_{n}(\alpha, \tau)$, for several of the inverse quantile regressions in the IVQR estimation. The point estimate is the value of $\alpha$ where the objective function is minimized, and the 95 percent confidence interval contains those values of $\alpha$ for which objective function is below the five percent critical value, which is also drawn in the plots. Panels A, B, and $\mathrm{C}$ show the objective function for estimating the effect of military service on log weekly wages of whites at the 25th, 80th, and 15 th percentile, respectively. The objective function for these panels appears to be close to globally convex, with a clear unique minimum, although at more extreme quantiles perhaps less so, as in Panel C. The estimator in Panel A is not significantly different from zero, while the estimates in Panels B and $\mathrm{C}$ are (marginally) significantly greater than and less than zero, respectively. As mentioned above, Panels A, B, and C exemplify that for well-conditioned, roughly continuous data such as log weekly wages, this approach is very tractable computationally.

However, as noted in the tables, when the data are discrete, or semi-discrete with mass points, the optimization problem in quantile regression is not well-behaved, and failed to converge in many cases. Panel D in Figure 5 shows the objective function for the effect of military service on wage and salary income for non-whites at the 55th percentile. This variable tended to have mass points, and the objective function is not nearly as well-behaved as for log weekly wages, with numerous local minima. The estimation illustrated in Panel D converged and yielded a reliable-seeming confidence interval, but as noted in the tables, many cases failed to converge. The education variable is even more problematic, and consequently I did not compute IVQR estimates for this outcome. Although I do not explore it further in this paper, Koenker and Hallock (2000) discuss some approaches to compute quantile regression estimates for discrete data, including a smoothing approach similar to Horowitz (1992). These approaches could ameliorate the computational problems exemplified in Panel C, and would be an interesting area for future research.

\section{Conclusion}

In this paper I examined the long term effects of military service in Vietnam on the distribution of several economic outcomes. OLS comparisons of whtie veterans and non-veterans show a significant negative relationship between military service and earnings and education. Conditional quantile regression enriches the picture, showing that while the association is negative across the distribution, it is much more so for the higher quantiles. This pattern can be explained either by a much stronger selection effect at the upper end of the distribution, or a long term causal effect of military service, 


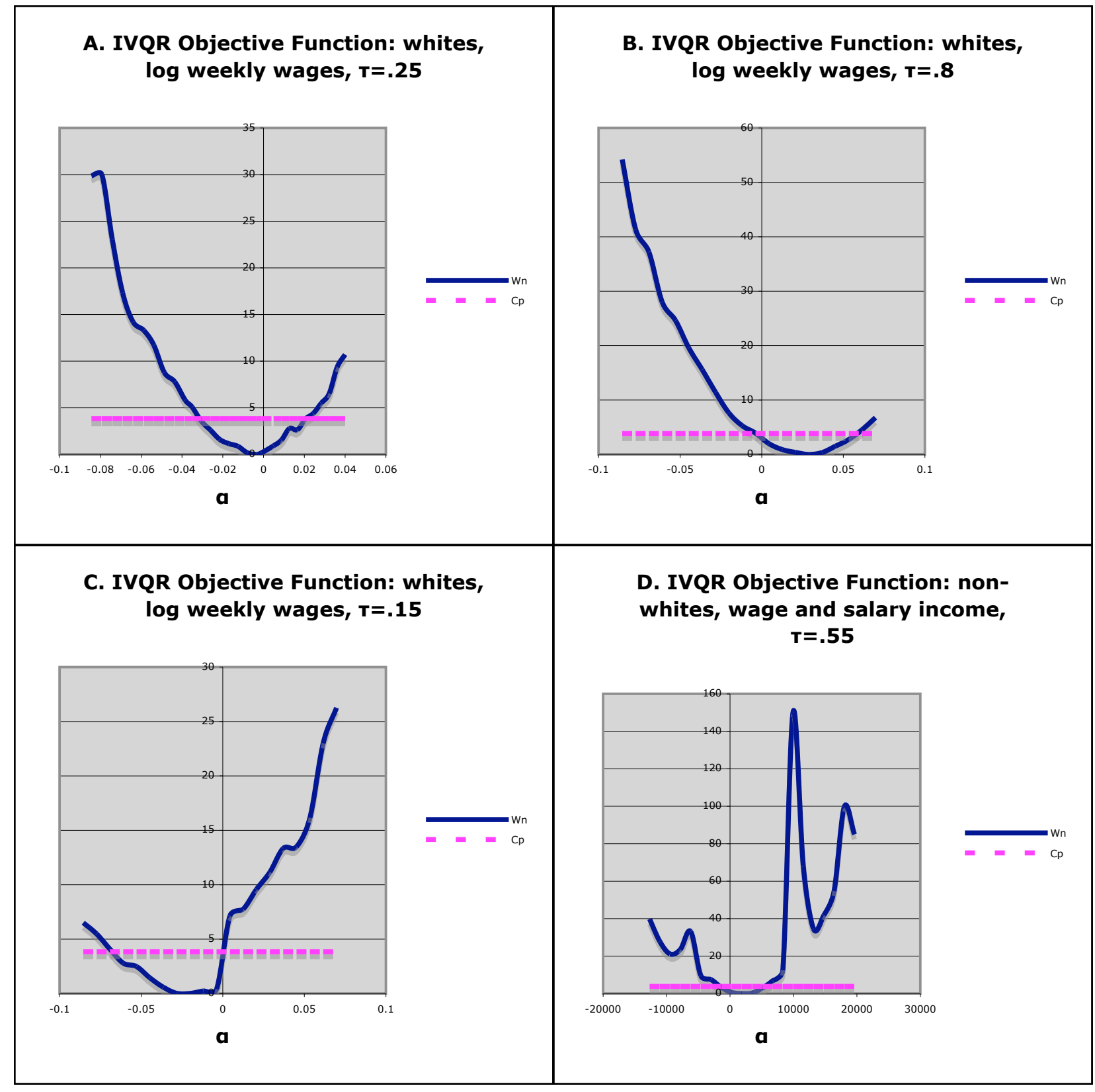

Figure 5: Objective functions and critical values for selected instrumental variables quantile regressions. 
such as a lasting loss-of-experience effect in a Mincer-type setting. I estimated the parameters of a causal quantile response function by two different methodologies to distinguish between these two explanations. The results of the quantile treatment effects estimation show that the pattern evident in the ordinary quantile regression is largely absent, and possibly even reversed, suggesting that heterogeneous selection effects, with stronger effects at the upper end of the distribution, are responsible for the conditional quantile patter. The causal effect of military service on the economic outcomes I looked at seems to be close to zero across the distribution, although there is some evidence of a lasting negative effect on log weekly wages for white men near the bottom of the distribution, and a positive effect at the top.

In addition to the empirical findings, my results offer a comparison between the LQTE estimator proposed by Abadie, Angrist, and Imbens (2002) and the IVQR estimator illustrated in Chernozhukov and Hansen (2008). The comparison constitutes an informal joint test of the validity of the assumptions required by each model and the external applicability of characteristics of the complier subpopulation. The similarity of the results across the two estimators suggests that the assumptions of each model hold for the data, and that looking at the compliers gives a fairly representative picture of the sample as a whole.

A possible question for future research is how the negative average effect found for white men's earnings within a decade or so of military service looks across the distribution. Performing the same exercise I did in this paper, but using the non-public 1980 US census file would allow a richer test of the hypothesis that loss of experience in a Mincer setting is the principle channel for the negative effect observed for white men.

Methodologically, an area of research would be to generalize the LQTE approach to allow for continuous treatments, or overidentification. Computationally, the difficulties the approaches had with discrete, or semi-discrete data make a smoothing approach such as Horowitz's (1992), or approaches discussed in Koenker and Hallock (2000) appealing areas for refinement of the estimators.

\section{References}

Abadie, Alberto (2000): "Semiparametric Estimation of Instrumental Variable Models for Causal Effects," NBER Technical Working Paper No. 260.

(2002): "Bootstrap Tests for Distributional Treatment Effects in Instrumental Variable Models," Journal of the American Statistical Association, 97, 284-292.

Abadie, Alberto, Joshua D. Angrist, and Guido Imbens (2002): "Instrumental Variables Estimates of the Effect of Subsidized Training on the Quantiles of Trainee Earnings," Econometrica, 70, 91-117.

Angrist, Joshua D. (1990): "Lifetime Earnings and the Vietnam Era Draft Lotter: Evidence from Social Security Administrative Records," American Economic Review, 80, 313-336.

Angrist, Joshua D., and Stacey Chen (2007): "Long-Term Effects of Vietnam-Era Conscription: Schooling, Experience and Earnings," NBER Working Paper No. 13411.

Angrist, Joshua D., Guido W. Imbens, and Donald B. Rubin (1996): "Identification of Causal Effects Using Instrumental Variables," Journal of the American Statistical Association, 91, 444-455.

Angrist, Joshua D., and Alan B. Krueger (1995): "Split-Sample Instrumental Variables Estimates of the Return to Schooling," Journal of Business 83 Economic Statistics, 13, 225-235.

Bassett, Gilbert, Jr., and Roger Koenker (1982): "An Empirical Quantile Function for Linear Models with |operatornameiid Errors," Journal of the American Statistical Association, $77,407-415$. 
Chernozhukov, Victor, and Christian Hansen (2005): "An IV Model of Quantile Treatment Effects," Econometrica, 73, 245-261.

(2008): "Instrumental Variable Quantile Regression: A Robust Inference Approach," Journal of Econometrics, 142, 379-398.

Horowitz, Joel L. (1992): "A Smoothed Maximum Score Estimator for the Binary Response Model," Econometrica, 60, 505-531.

Jaeger, David A. (1997): "Reconciling the Old and New Census Bureau Education Questions: Recommendations for Researchers," Journal of Business and Economic Statistics, 15, 300-309.

Koenker, Roger, and Jr. Bassett, Gilbert (1978): "Regression Quantiles," Econometrica, $46,33-50$.

Koenker, Roger, and Kevin Hallock (2000): "Quantile REgression: An Introduction," Available at http://www.econ.uiuc.edu/ roger/research/intro/intro.html.

Mallows, C. L. (1973): "Some Comments on $C_{P}$," Technometrics, 15, 661-675.

Mincer, Jacob (1974): Schooling, Experience, and Earnings. National Bureau of Economic Research, New York.

Oi, Walter Y. (1967): "The Economic Cost of the Draft," American Economic Review, 57, 39-62.

Rangel, Charles B. (2007): "Universal National Service Act of 2007 (Introduced in House)," H.R. 393, Retrieved on January 28, 2008 from http://thomas.loc.gov.

— (December 31, 2002): "Bring Back the Draft," New York Times.

Rosen, Sherwin, and Paul Taubman (1982): "Changes in Life-Cycle Earnings: What Do Social Security Data Show?," The Journal of Human Resources, 17, 321-338.

Seltzer, Carl C., and Seymour Jablon (1974): "Effects of Selection on Mortality," American Journal of Epidemiology, 100.

The Wall Street Journal (November 25, 2006): "Uncle Charlie Wants You! The Draft Would Weaken the World's Best Military," The Wall Street Journal. 